•综述・

\title{
蚳蚓调控土壤微生态缓解连作障碍的作用机制
}

\author{
毕艳孟 1,2 孙振钧 ${ }^{1 *}$ \\ 1 (中国农业大学资源与环境学院, 北京 100193) \\ 2 (中国医学科学院北京协和医学院药用植物研究所, 北京 100193)
}

\begin{abstract}
摘要: 连作障碍不仅严重影响作物产量, 而且会导致土壤生物多样性下降、有益微生物减少及病原菌增加等一系 列微生态失衡问题。土壤微生态失衡反作用于植物, 导致植物发生更严重的病害、减产等。作为土壤生态系统工 程师, 虾蚂的取食、掘洞和爬行等活动对土壤微生态具有重要的调控作用, 既可以改善土壤环境, 又可以强化土壤 生物群落功能, 有望为缓解作物的连作障碍问题提供一条新途径。本文总结了土壤微生态与土壤功能维持及蚯蚂 调控土壤生物作用的研究进展, 在此基础上, 结合蝶蚓对化感物质降解作用的研究, 分析了虾蚂通过调控土壤微 生态缓解作物连作障碍的微生物作用机制的三条途径: 直接调控微生物群落、通过改变化感物质组成以及通过调 控土壤动物区系调控微生物群落。蛏蚂对微生物群落的调控可改善失衡的土壤根际微生态, 有效缓解作物连作障碍。
\end{abstract} 关键词: 虾蚂; 连作障碍; 土壤动物; 化感物质; 土壤微生物

\section{Mechanisms of earthworms to alleviate continuous cropping obstacles through regulating soil microecology}

\author{
Yanmeng $\mathrm{Bi}^{1,2}$, Zhenjun Sun ${ }^{{ }^{*}}$ \\ 1 College of Resources and Environmental Science, China Agricultural University, Beijing 100193 \\ 2 Institute of Medicinal Plant Development, Chinese Academy of Medical Sciences and Beijing Union Medical College, \\ Beijing 100193
}

\begin{abstract}
Obstacles to continuous cropping seriously affect crop yield and also lead to a series of microecological imbalance problems, such as the decline of soil biodiversity, the decrease of beneficial microbes, and the increase of pathogens. The imbalanced soil microecology affects plants and can lead to more serious disease and even greater reduction of crop yield. As soil ecosystem engineers, earthworms have important regulating effects on soil microecology. Earthworms can improve the soil environment and strengthen the function of soil biological communities through their activities (e.g. feeding, burrowing and creeping), thus providing a potential way to alleviate microecological obstacles to continuous cropping. This paper reviews the progress made in research on soil microecology, soil function maintenance, and earthworm regulation of soil biological function. Three mechanisms that earthworms employ to alleviate obstacles to continuous cropping through soil microecology regulation were identified: direct regulation of soil microbial communities, regulation of microbial colonies by changing allelochemical composition, and regulation of soil fauna communities. Through earthworm regulation of microbial communities, an imbalanced soil microenvironment in the rhizosphere is corrected, thereby alleviating obstacles to continuous cropping.
\end{abstract}

Key words: earthworms; obstacles to continuous cropping; soil animal; allelochemicals; soil microbes

植物通过地上与地下部的物质交换持续影响 其生存环境, 环境的改变则反作用于植物, 影响植 物的生长和发育(Kulmatiski et al, 2008)。在自然生 态系统中, 土壤-植物的反馈作用是局域尺度保持
植物多样性的重要驱动力量, 土壤生物的综合作用 不利于同种或者近缘种在相同的地点生长繁殖 (Huang et al, 2013b; Teste et al, 2017)。然而, 在农业 生产中, 随着耕地面积的减少、设施栽培的发展以 
及某些作物对特定气候和土壤的要求, 在同一地块 进行作物再植的现象普遍存在, 导致连作障碍发 生。连作障碍使作物生长发育不良、病害加重、品 质下降，成为限制农业可持续发展的瓶颈。从土壤 的角度讲，作物连作障碍导致土壤微生态系统紊乱， 土壤生物多样性降低, 生态系统稳定性下降。

生产上用来克服连作障碍的措施主要是土壤 灭菌, 该方法虽然在一定程度上缓解了连作障碍, 但从长远看, 对环境和土壤造成的危害是巨大且深 远的。因此, 人们也在探索一些环境友好的方法, 希望通过调控土壤生态的方式缓解作物连作障碍。 土壤生态的调控方式主要有两种: 一种是调控环境, 如施用某些土壤添加剂, 改变土壤的理化性质, 间 接影响土壤生物; 一种是调控生物, 如接种某些具 有拮抗功能的微生物, 改变土壤微生物群落结构, 间接影响土壤环境。相对于简单的土壤灭菌而言, 调控土壤微生态的方式更符合整体、协调、循环、 再生的原则, 有利于农业的可持续发展。

虾蚓是土壤生态系统中的大型无脊椎动物, 由 于其对土壤生态系统具有重要的调控作用, 被誉为 “土壤生态系统工程师” (Jones et al, 1994)。通常蚳 蚓被认为能够促进植物的生长(Lee, 1985; Edwards \& Bohlen, 1996), 而且其数量常被人们用作表征土 壤健康的重要指标(Doran \& Zeiss, 2000)。虾蚓对土 壤环境和土壤生物的调控作用已有大量报道, 然而 很少有研究关注蚟蚓对作物连作障碍的防控作用。 本文通过分析连作障碍发生的生态学机理, 总结了 蝶蚓对土壤微生态的调控作用, 以期为缓解作物连 作障碍问题提供新的思路。

\section{土壤微生态平衡}

\section{1 微生态平衡与健康土壤功能的维持}

健康土壤是能够持续提供生物产品, 保障空气 质量和水源安全, 对植物、动物和人类健康发挥重 要作用的生命系统(Doran et al, 1996)。土壤微生物 是维持土壤中各种生物化学过程动态平衡的核心, 它在矿质养分循环、有机质更新、土壤结构及土壤 肥力形成过程中发挥着重要作用(Brussaard et al, 2007; Lambers et al, 2009)。冊庸置疑, 土壤微生物 多样性是决定土壤健康与否的重要因素之一, 并且 是保持土壤抗病性的重要驱动力 (Garbeva et al, 2004)。一般来说, 土壤微生物多样性与土壤生物群
落结构的复杂程度关系很大，一个微生物多样性较 高的土壤生态系统, 调节能力较强, 稳定性就越高, 土壤健康状况就越好(He et al, 2009; Nannipieri et al, 2010; Larkin, 2015)。决定土壤微生态平衡的关键在 于土壤微生物与植物、土壤动物之间的相互作用 (Garbeva et al, 2004; 陈小云等, 2007; Watkins et al, 2009)。在一个稳定的土壤生态系统中，土壤微生物 与植物和土壤动物之间相互作用、各种生物类群之 间的相互制约和相互依存关系使得整个土壤微生 态系统处于稳定的状态, 即土壤微生态平衡。

\section{2 土壤生物间的相互关系}

\subsection{1 植物与土壤微生物的关系}

土壤微生物的生存和繁殖依赖于从土壤中获 得的碳源(Garbeva et al, 2004), 而土壤中的碳主要 源于植物的光合产物(Bais et al, 2006), 因此, 根际 区土壤微生物的密度远远大于非根际区土壤(Berg et al, 2006)。根据对植物的作用，可以大体将植物根 际微生物分为三类。第一类是根际促生菌，这类微 生物能够通过自身分泌物促进植物根系的生长, 或 者通过诱导植物根系启动自身的免疫系统以抵御 病原菌入侵，还能通过自身作用在相邻的植株之间 进行信号传导(Song et al, 2010; Chung et al, 2015; Kim et al, 2015)。第二类是病原菌，它们可以分泌一 些毒素抑制植物的生长, 或者直接侵入植物体内, 引发植物病害(Koike et al, 2009; Fang et al, 2012)。 第三类是依赖植物资源但对植物没有直接反馈作 用的微生物, 这也是根际区种类和数量最多的一类 微生物(Berendsen et al, 2012)。除了对植物的作用, 这三类微生物之间也会相互促进或抑制(Toljander et al, 2007), 而植物除了为根际区微生物提供碳源 以外, 还会分泌一些次级代谢产物(或者植物残体 腐解产生的物质), 这些物质进入土壤, 对微生物有 刺激或者抑制作用。植物对微生物、微生物对植物 以及微生物之间都存在着相互作用(图1)。

\subsection{2 植物与土壤动物的关系}

植物作为生产者, 是土壤生态系统中能量的主 要来源。一方面, 植物的根系为某些植食性线虫、 昆虫提供了食物(Oka, 2010); 另一方面, 植物地上 部分调落后进入土壤, 为腐食性动物提供了生存和 繁殖的能源(张雪萍等, 2001)。此外, 植物还可以通 过根系分泌物影响土壤动物，如草莓分泌的对羟基 苯甲酸和对香豆酸可以使土壤中线虫总量和食细 
菌线虫数量增加(李贺勤等, 2014), 而这些以植物或 碎屑为食的小型动物会被体型更大的捕食性土壤 动物如捕食性线虫、螨类、跳虫等取食(Moore et al, 1990), 从而使植物固定的能量流入动物。而动物的 活动对植物的生长和抗病也有重要的作用(Schrader et al, 2013): 土壤动物可以通过直接取食作用来破 碎植物地上部分的枯落物, 或者通过影响周围的理 化环境、协同微生物的降解作用，间接影响枯落物 的分解, 从而促进土壤生态系统中物质循环, 为植 物提供必要的营养元素(González \& Seastedt, 2001; Zhang et al, 2018)。另外, 某些土壤动物还可以通过 与植物地下部分作用, 诱导植物自身的抗虫、抗病的 防御反应，减少病虫害的发生(Blouin et al, 2005)。

\subsection{3 土壤动物与微生物的关系}

许多土壤中细菌和真菌是土壤动物食物的主 要来源, 土壤中的食微动物(如某些线虫、螨类、原 生动物、小型节肢动物等)通过取食细菌、真菌获得 能量, 是外界能量输入土壤生态系统的重要途径 (Sauvadet et al, 2016)。食细菌和食真菌的线虫可以 通过取食作用调控微生物群落组成、结构和代谢活 性，并释放固持在微生物中的养分 (吴纪华等, 2007)。原生动物可以进入到较小的土壤空隙中捕食 细菌, 调节细菌群落结构(Corno \& Jürgens, 2006)。

除捕食作用以外，土壤动物的活动还可以提高 土壤养分的矿化率，从而影响土壤微生物量和群落 结构(朱永恒等, 2012)。土壤动物类群之间也会通过

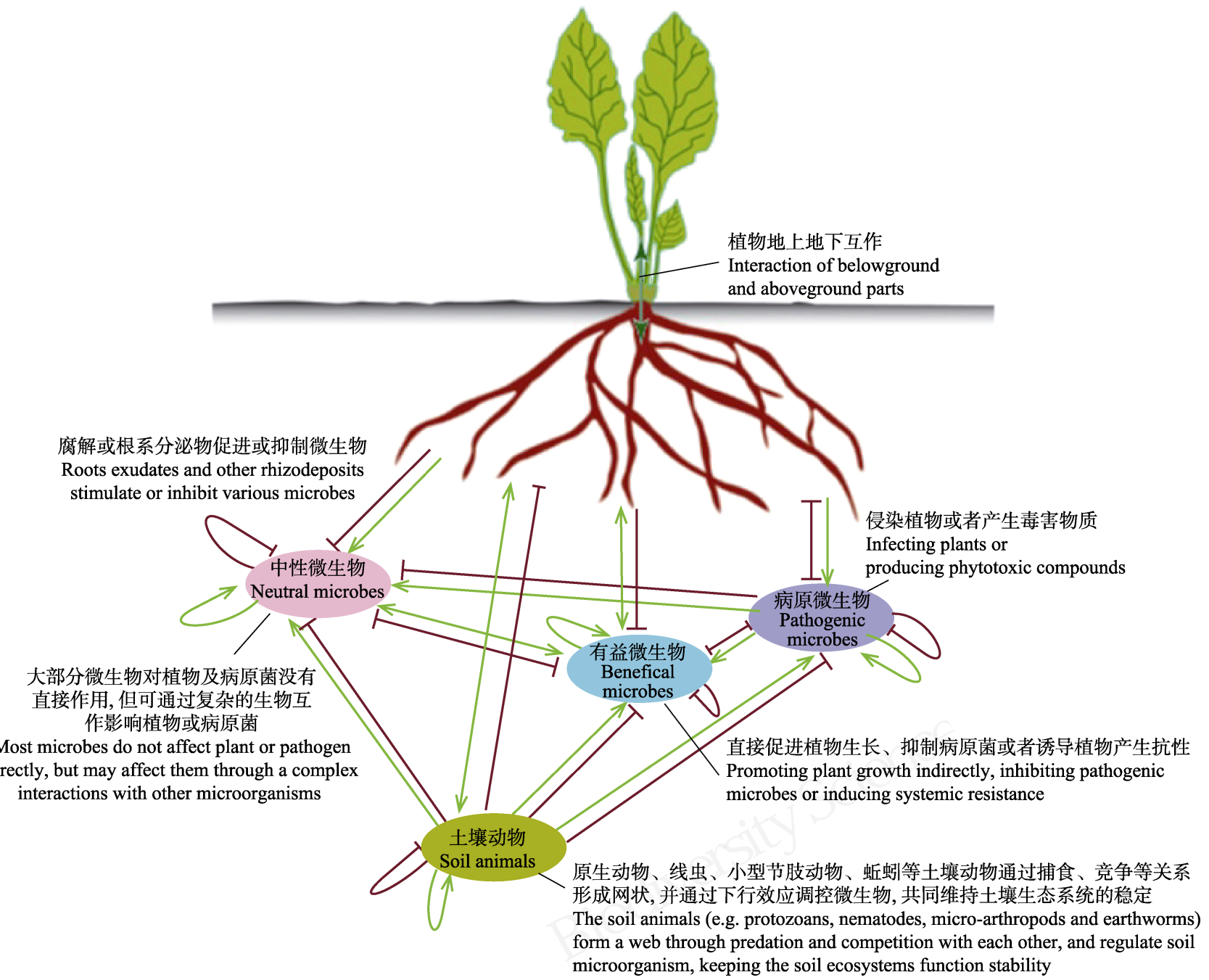

图1 基于各功能群相互作用的土壤微生态平衡(仿自Berendsen et al, 2012)。其中绿线加箭头表示促进作用, 红线加短线表示 抑制作用。

Fig. 1 The balance of ecosystem (based on the interaction of functional groups) (modified from Berendsen et al, 2012). Green line indicates promoting effect and red line indicates inhibiting effect. 
相互作用，依靠复杂的反馈和负反馈机制维持土壤 动物群落组成的相对稳定(Geisen et al, 2016; 杜晓 芳等, 2018)。

综上所述, 土壤中植物根系、微生物、动物之 间的作用主要是通过“物流”联系起来的：一种“物 流” 是通过取食与被食关系形成的食物链, 主要体 现在植物与动物、微生物与动物以及动物与动物之 间的直接作用上; 另一种是非食物链“物流”, 即生 物之间并不直接接触，而是通过分泌或者腐解产生 的物质建立联系, 主要体现在植物与微生物、微生 物与微生物之间的间接作用上。生物之间的这些作 用, 既有促进, 也有抑制, 其方向和强度是通过系 统内部信息传递控制的。完善的信息传递系统使得 系统内部各组分保持相对稳定的状态。在健康土壤 中, 植物病原菌因受到土壤生态系统内部各种作用 的制约, 种群不会无限制扩大, 就不会引起植物大 规模病害的暴发(Janvier et al, 2007)。因此, 在植物 地下部所处的土壤中, 各种生物通过食物链与非食 物链物流相互作用, 交织形成网状结构, 生态系统 完善的信息传递功能使这张网有条不紊地发挥作 用(图1)。

\section{蚟蚂对土壤生物的调控作用}

\section{1 蚯蚓对土壤动物的调控作用}

土壤动物对土壤生态系统功能的维持具有重 要作用。根据体宽, 土壤动物可以分为小型 (microfauna, 平均体宽小于 0.1 或 $0.2 \mathrm{~mm}$ )、中型 (mesofauna, 平均体宽在0.1或0.2-2 mm之间)、大型 (macrofauna, 平均体宽大于 $2 \mathrm{~mm}$ )及巨型土壤动物 (megafauna, 平均体宽大于 $2 \mathrm{~cm}$ ) (Wurst et al, 2013)。蝶蚓作为土壤中的大型动物, 可以通过下行 效应调控其他中型和小型动物。

以威廉腔蚓(Metaphire guillelmi)为例, Tao等 (2011)研究表明, 虾蚓可以取食线虫, 导致线虫丰 富度下降。Boyer等(2013)以南美暗蚂(Pontoscolex corethrurus)为材料, 同样发现虾蚓可以取食线虫, 并能够降低土壤中线虫囊泡和卵的数量。 Ilieva-Makulec和Makulec (2002)研究表明, 接种粉 正蚂(Lumbricus rubellus) 30天后, 食细菌、食真菌 以及植食性线虫丰富度均表现出降低的趋势。除了 直接取食外，蚯蚓的掘洞等行为也会影响线虫的生 活环境, 改变它们在不同土层的分布情况(Tao et al,
2009; Andriuzzi et al, 2016)。

另外，也有相关研究报道了蚯蚓对螨类和原生 动物等土壤动物的调控情况。Mclean和Parkinson (1998)利用中宇宙系统(mesocosm)模拟了表居型 (epigeic)八毛枝蚓(Dendrobaena octaedra)对甲螨群 落的影响, 研究表明, 接种虾蚓 3 个月后甲螨类多 样性升高, 6 个月后其丰富度也显著提高。本课题组 前期研究表明，接种赤子爱胜蚓(Eisenia fetida) 6 个 月后土壤中螨类各亚目的丰富度显著提高(伍玉鹏 等, 2013)。Bonkowski和Schaefer (1997)研究表明, 流蚓属(Aporrectodea)的A. caliginos可以取食自由活 动的原生动物, 对原生动物的分布有重要的影响。

此外, 蚯蚓与土壤跳虫关系密切, 但是由于研 究者所关注的蚟蚓和跳虫种的不同, 得出的结论是 多样的。Marinissen和Bok (1998)研究发现, 蚯蚓的 活动增加了土壤的孔隙度, 从而使得跳虫数目和种 类增加; Salmon和Ponge (2001)研究发现流蚓属的 蚯蚓 Aporrectodea giardi 及绿色异唇蚓 (Allolobophora chlorotica) 的活动可以增加跳虫 Heteromurus nitidus的数量, 并分析原因是蛏蚓分泌的粘 液以及排出的尿液可以强烈吸引这类跳虫; 而Gao 等(2017)研究却表明, 远盲蚓属(Amynthas)的蛲蚓A. agrestis入侵后，造成曲毛裸长跳虫(Sinella curviseta) 数量减少。

蚯蚓活动对主要土壤动物类群的调控作用影 响了土壤食物网功能的发挥, 进而会间接影响土壤 微生物的群落。

\section{2 蚯蚓对土壤微生物的调控作用}

蚯蚓和微生物的关系是非常复杂的, 微生物是 虾蚓营养的主要来源, 同时虾蚓通过与微生物共同 作用降解有机物质的过程促进微生物活性的提高 (Li et al, 2002), 并且蚯蚓可以通过自身活动协助微 生物在土壤中传播(Edwards \& Bohlen, 1996)。虾蚓 可以通过改善微生境(排粪、作穴、搅动)、提高有 机物的表面积、直接取食、携带传播微生物等方式 影响土壤微生物数量、活性、组成和功能, 还可以 通过改变土壤结构来加速土壤营养物质的循环，影 响微生物的群落结构(Edwards \& Fletcher，1988; Natal- da-Luz et al, 2012; Akca et al, 2014)。

\subsection{1 影响微生物量、活性和多样性}

土壤微生物量是土壤重要的源和汇，对土壤养 分循环和植物生长具有重要作用(Singh et al, 1989), 
大量研究报道蚳蚓活动会影响土壤微生物量。 Burtelow等(1998)在美国东北部森林中研究了夏威 远盲蚂(Amynthas hawayanus)入侵对土壤微生物的 影响, 发现蚯蚓入侵后会形成明显的斑块(patches), 斑块内部土壤微生物量碳、氮显著提高。 $\mathrm{Li}$ 等(2002) 发现纽约中部阔叶林中蚯蚓的入侵能够显著提高 土壤微生物量。姚影等(2015)利用微宇宙实验研究 了虾蚓对施入秸秆后土壤有机碳的影响, 结果表明 赤子爱胜蚓能提高土壤微生物量。伍玉鹏等(2013) 在探索蛏蚓改良盐碱地的作用时, 也发现接种赤子 爱胜蚓能显著提高土壤微生物量。Bi等(2018)在研 究虹蚓对草莓枯病的抑制作用时, 同样发现赤子爱 胜蚓和威廉腔蚓能提高土壤微生物量。

蚯蚂对微生物量的影响作用可能与肠道厌氧 环境有关, Drake和Horn (2007)发现蝶蚓肠道内可培 养厌氧细菌数量最高能达到未被取食土壤的 4,000 倍。但是也有研究表明虾蚓活动会降低土壤微生物 量。Eisenhauer等(2011)研究了蛏蚓入侵北美北部阔 叶林的过程, 发现蛏蚓可使土壤微生物量降低达 42\%。Gomez-Brandon等(2011)在研究安德爱胜蚓 (Eisenia andrei)消化道的作用时, 发现安德爱胜蚓 粪便中的土壤微生物量低于其食物中的量。Zhang 等(2000)向土壤中接种赤子爱胜蚓和威廉腔蚓, 处 理土壤24 h 后发现, 两种虾蚓均会显著降低土壤微 生物量。

土壤微生物活性对植物生长有重要作用, 一般 用土壤呼吸和土壤酶活性进行表征。多数研究表明 蝶蚓具有提高土壤微生物活性的作用。在对纽约州 一阔叶林蚯蚓入侵的研究中, Li等(2002)发现, 虾蚓 可使土壤基础呼吸提高5倍, 底物诱导呼吸提高 6.7 倍, 土壤微生物代谢熵提高3倍。Schaefer等(2005) 研究了石油泄漏污染的土壤中接种陆正蚂 (Lumbricus terrestris)、绿色异唇蚓及赤子爱胜蚓28 天后，土壤呼吸作用均显著增强，而不接种蝶蚓的 土壤呼吸则无显著变化; 同时研究者观察到, 赤子 爱胜蚓与陆正蚓对土壤呼吸作用的影响大于绿色 异唇蚓。Barbosa等(2017)发现蚯蚓提高了土壤呼吸 和微生物量, 促进了菜豆(Phaseolus vulgaris)的生 长。Cao等(2016)在研究土霉素污染的土壤中蛏蚂对 养分循环的作用时发现, 接种赤子爱胜蚓的土壤嫝 酶活性显著提高。Zhang等(2016)研究表明, 在轻度 盐碱胁迫 $(\mathrm{EC}=4.41 \mathrm{mS} / \mathrm{cm})$ 的土壤中接种赤子爱胜
蚓, 土壤过氧化氢酶活性显著提高。本课题组研究 了虾蚓在连续 100 天对植物残体产生的酚类物质降 解过程的影响, 发现虾蚓的活动加速了酚类物质降 解过程中微生物群落的演替, 而且接种蝶蚓的处理 组中土壤脱氢酶活性高于同一时期取样的对照组 (毕艳孟，2016)。Huang等(2013a)发现，在处理果蔬 废弃物的过程中, 无论接种成熟的赤子爱胜蚓还是 其幼蚓，土壤脱氢酶活性均显著低于对照处理。李 欢等(2016)研究虹蚓菌根对土壤酶活和甘薯根系生 长的作用时, 发现接种蝶蚓可以显著提高土壤脲酶 和碱性磷酸酶活性，从而促进甘薯的生长。

针对蚯蚓对土壤微生物多样性的作用也有大 量研究。Lipiec等(2016)研究了蝶蚓打洞行为对微生 物的影响, 发现蚯蚓洞穴土壤中平均颜色变化率 (averagewell color development, 表征微生物对碳源 利用的指标)显著高于周围土壤。郑宪清等(2015)利 用定位实验, 研究了威廉腔蚓对土壤微生物多样性 的影响，发现投放蚯蚓后，土壤微生物的Simpson 和Shannon-Wiener多样性指数显著高于对照组。代 金君等(2015)研究了虾蚓肠道对重金属污染土壤微 生物群落的影响, 发现经过蛏蚓过腹作用, 土壤微 生物Shannon-Wiener多样性指数显著高于未经蛏蚓 取食的土壤, 且赤子爱胜蚓和壮伟远盲蚓 (Amynthas robustu)对微生物多样性的作用无显著性差 异。Zhang等(2013)也研究了赤子爱胜蚓肠道对微生 物群落的作用, 发现细菌在虾蚓肠道中多样性先增 加后减少，最后保持稳定的状态。

蚯蚓对土壤微生物的作用是复杂和多样的, 不 同的研究可能得出不同甚至相反的结论，这可能与 研究者选取土壤的性质、接种蚯蚓的密度以及选用 蚯蚓的生活型及种属差异等因素有关。但是这些因 素如何影响虹蚂对微生物的调控作用及其作用机 理等问题, 仍然需要系统深入的研究。

\subsection{2 改变微生物群落结构}

蝶蚓对微生物调控作用的研究除集中于对微 生物总体生物量、活性、多样性的影响以外, 还有 许多关注了蝶蚓对微生物群落结构的调控。

蝶蚓的取食偏好是改变微生物群落结构的重 要因素。Shan等(2013)利用同位素标记研究了威廉 腔蚓食物的主要来源，证实相对细菌而言，该蚯蚓 更喜欢取食真菌。除了虾蚓的取食偏好, 其肠道对 微生物群落的塑造作用也能够改变不同微生物所 
占比例。Chapuis-Lardy等(2010)在研究蚯蚓对于土 壤中 $\mathrm{CO}_{2}$ 和 $\mathrm{N}_{2} \mathrm{O}$ 释放的作用时发现, 被南美岸蚓 (Pontoscolex corethrurus)取食并排泄后的土壤中真 菌总量相对于未被取食的土壤显著下降。蝶蚓肠道 对不同类型的土壤微生物有不同的影响，真菌大部 分被杀死, 而生长较快的细菌会迅速生长繁殖并进 入有机质含量更高的蛏蚓粪中 (Thakuria et al, 2010)。另外, 蚳蚓对土壤环境的改变也是导致细菌 /真菌比例变化的原因。Dempsey等(2011)通过仲夏 和初秋时期对阔叶林土壤的调查发现, 蚯蚓能够显 著提高细菌真菌比例, 并推测这与蚯蚓活动改变土 壤有机质层有关。

许多研究表明, 蝶蚓活动可以直接减少病原菌 的数量。Wolfarth 等(2011) 利用流蚓属虾蚓 Aporrectodea caliginosa结合覆盖秸秆残渣, 显著降低 了镰刀菌属(Fusarium)的生物量和镰刀菌酸的浓度; 类似地, Oldenburg等(2008)在利用陆正蚓降解小麦 秸秆的过程中发现, 该蚳蚓能够降低镰刀菌的生物 量和镰刀菌酸的浓度。Elmer和Ferrandino (2009)通 过挖掘沟壑的方式调控田间蛏蚓种群数量, 发现蝶 蚓种群的增大有利于减少茄子种植土壤中大丽轮 枝菌(Verticillium dahlia)的数量。Bonkowski等(2000) 通过对5种蛏蚓取食真菌偏好的研究发现, 蝐蚓可 以取食镰刀菌、丝核菌等植物病原菌。

也有研究表明, 蝶蚓通过提高根际促生菌及病 原拮抗菌的数量, 间接促进植物的生长, 抑制病害 发生。Wu等(2012)研究了威廉环毛蚓(Pheretima guillelmi, 即上文提到的威廉腔蚓)和根际促生菌的 协同作用，发现该虾蚓能够显著提高Azotobacter chroococcum、Bacillus megaterium 和B. mucilaginous 三种根际促生菌的丰度, 进而强化了细菌加速养分 循环的作用。Elmer (2009)系统研究了陆正蚓对芦 笋、茄子和番茄病害的作用, 发现蝶蚓的引入提高 了土壤中荧光假单胞菌及丝状放线菌等有益菌群 的密度, 从而有效抑制了三种蔬菜病害的发生。除 了关于蝶蚓增加土壤中有益微生物的报道, 还有研 究直接从蚯蚓粪中获得了病原拮抗菌。Gopalakrishnan等(2011)从以植物秸秆为原料的虹蚓粪中分 离获得 5 株对镰刀菌有拮抗作用的细菌, 能够有效 防控鹰嘴豆的枯萎病。本课题组从以牛粪为原料的 蚯蚓粪中分离获得对植物病原菌具有显著抑制作 用的球孢链霉菌(Streptomyces globisporus)和丁香苷
链霉菌(S. syringini), 并将该蚯蚓粪施入黄瓜种植 土壤，显著降低了黄瓜苗期的立枯病和枯萎病的病 情指数(胡艳霞等, 2002)。

\section{蝶蚓对缓解连作障碍的作用}

\section{1 连作导致微生态失衡}

不同植物的根系分泌物或者残体腐解产生的 物质是不同的，由此它们对土壤生物尤其是微生物 群落有不同的选择作用(Zhang et al, 2014; Abbasi et al, 2015; Vaitauskiene et al, 2015), 使得土壤微生物 群落及其作用的方式呈现出多样化。然而, 在农田 连作的环境中，作物种类单一使得植物根系分泌或 者残体腐解产生的物质趋向于单一化，定向选择某 些根际区的微生物，导致根际区微生物多样性降低, 使系统信息传递功能以及系统内部生物之间的相 互促进和制约关系, 甚至整个土壤生态系统功能遭 到破坏。主要表现为微生物的活性和多样性降低 (Liu et al, 2014)、细菌/真菌比例下降(甄文超等, 2005; Li et al, 2014)、病原菌增加(Ye et al, 2004; Niu et al, 2011)。大多数土传病原菌既能营腐生生活, 又 能营寄生生活，一般会在根系区生长并繁殖到一定 的数量再侵染宿主细胞, 或者在宿主内扩繁到足够 的数量才能有效地侵染宿主组织(Berendsen et al, 2012)。而根际区失衡的微生物群落环境恰好为这些 病原菌的繁殖提供了条件，导致植物病害发生。根 际微生物群落失衡, 除了表现为病原菌增加外, 还 体现在有益微生物的减少上。许多微生物在土壤营 养循环中发挥着重要作用(Gyaneshwar et al, 2003; Jing et al, 2017), 因此, 功能紊乱的微生物群落可 能影响土壤养分的循环, 限制植物对营养的利用, 造成作物生长不良、病害加剧。

\section{2 蚯蚓促进化感物质降解}

作为土壤生态系统中的分解者, 蚟蚓的活动能 够促进土壤中许多物质降解，其中一些物质是对植 物或土壤微生物有重要调节作用的化感物质。酚类 物质就是一类重要的化感物质, 其在连作土壤中的 累积常被认为是造成作物连作障碍的原因之一(吴 宗伟等，2009; 李贺勤等，2014)。Butenschoen等 (2009)利用同位素标记的方法研究了土居型神女辛 石蚓(Octolasion tyrtaeum)对儿茶酚的作用，结果表 明，该蛏蚓能够通过提高微生物活性影响儿茶酚的 矿化过程。Bi等(2016)研究表明，接种表居型赤子爱 


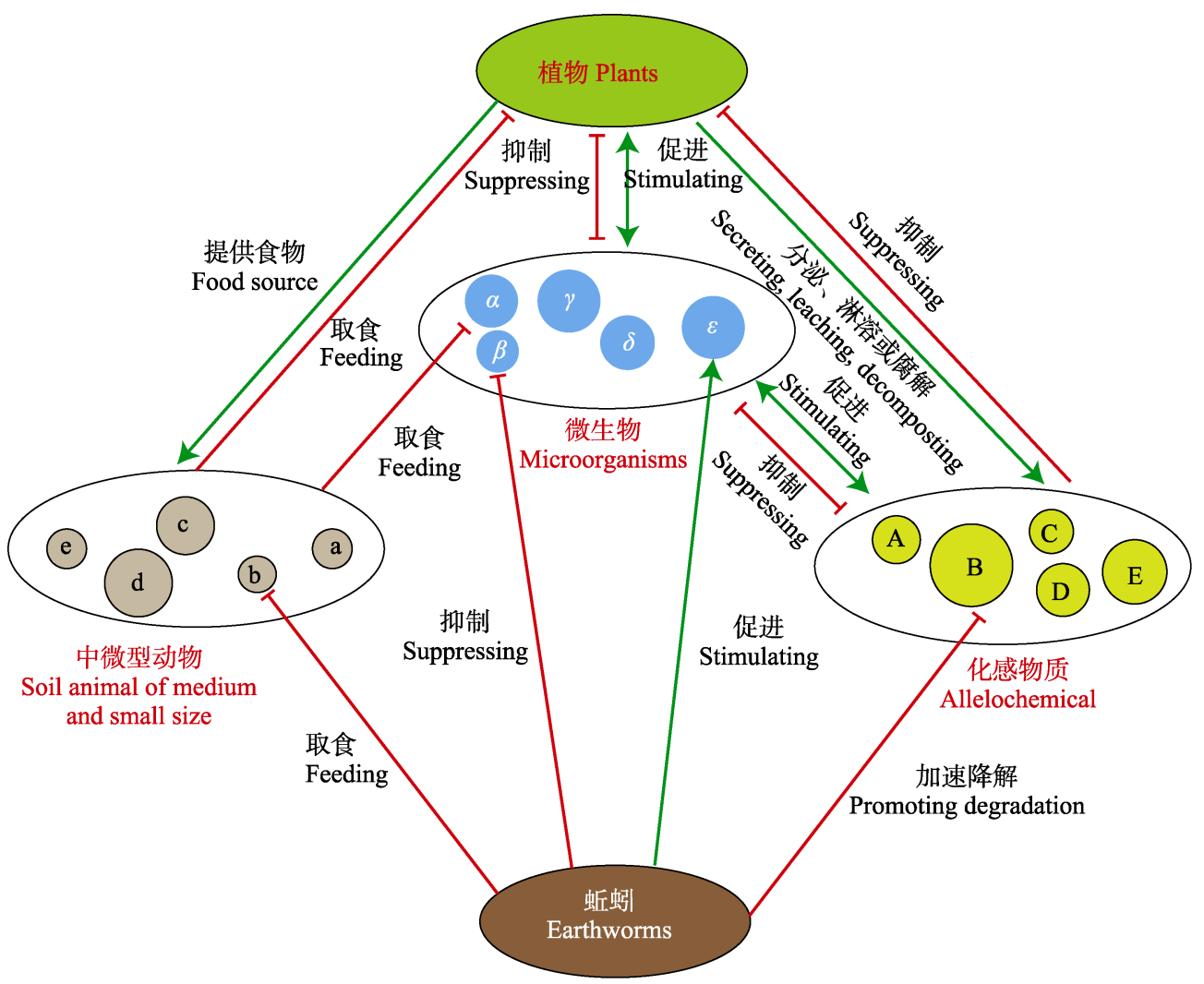

图2 蚯蚓调控连作障碍的土壤微生态机制

Fig. 2 The mechanism of earthworm regulating soil microecology

胜蚓和上食下居型威廉腔蚓均能加速酚类物质的 降解, 且降解过程中需要微生物的参与。Yahaya等 (2017) 研究发现, 赤子爱胜蚓和枝蚓属(Dendrobaena)的D. veneta处理果园内枯枝落叶, 能加速木 质素的降解，使得酚类物质含量显著降低。Mohan 等(2011) 评价了 3 种蚯蚓 (赤子爱胜蚓, Eudrillus eugenia和Anantapur species)对苯酚的耐受和降解能 力, 发现赤子爱胜蚓耐受能力最强, 可以耐受 100 $\mathrm{ppm}$ 的苯酚, 并在72 h内对该浓度的苯酚降解率达 $100 \%$ 。在研究蛏蚓对草莓枯萎病的防控效果时, $\mathrm{Bi}$ 等(2018)发现威廉腔蚓可以加速酚酸的降解, 且对 原儿茶酸和对香豆酸的降解效果优于其他酚酸, 并 分析该作用可能会降低草莓枯萎病病情指数。本课 题组在对西瓜连作的研究中还发现, 新鲜虹蚓粪对 香豆酸和丁香酸的降解能力较强, 由此可以改变土 壤中不同种类酚酸的比例(许永利, 2006) ${ }^{\circledR}$ 。除了酚 类化感物质以外, 也有研究表明虾蚓的活动可以改 变土壤中不同脂肪酸类物质的比例 (Sampedro \&

(1)许永利 (2006) 蛏蚂粪解除西瓜连作障碍的效果及机理初探. 硕士学 位论文, 中国农业大学, 北京.
Whalen, 2007)。目前尚未见有关蚯蚓对其他类化感 物质降解的报道。

\section{3 蚯蚓调控连作障碍土壤微生态机制}

在正常的土壤系统中, 由于地上部分植物是多 样的, 其分泌、淋溶或腐解产生的化感物质与土壤 微生物、土壤动物以及植物根系之间具有相互促进 和抑制作用，共同作用的结果使土壤生态系统处于 平衡态, 土壤能够稳定地发挥功能。但是, 连作导 致地上部分植物长期单一化, 使得土壤中化感物质 不均衡累积, 多样性下降; 同时由于单一植物对土 壤动物的选择, 使得土壤动物类群定向改变, 造成 动物区系平衡被打破, 多样性下降, 功能紊乱; 植 物的单一化还会导致土壤微生物群落的组成类群 定向改变, 微生物区系平衡被打破。增加的化感物 质以及功能紊乱的动物区系进一步加剧微生物区 系的失衡, 造成生态系统稳定性下降, 系统原本具 有的抑制病原菌的能力降低, 导致植物病原菌数量 增加, 作物病害发生。

蚳蚓能够直接调控微生物, 影响微生物的总 量、活性和多样性, 改变微生物的比例(Li et al, 2002; 
Wu et al, 2012; 郑宪清等, 2015; Barbosa et al, 2017)。另外, 蚯蚓作为生态系统的分解者, 能够加 速化感物质的降解, 而且可能改变土壤中化感物质 的比例, 调整失衡的化感物质(Bi et al, 2018)。同时, 蚟蚓作为土壤生态系统的高级消费者, 能够取食某 些中、小型土壤动物, 通过下行效应调控土壤动物 区系，提高土壤动物的多样性(Mclean \& Parkinson, 1998; 伍玉鹏等, 2013)。化感物质多样性和动物多 样性的恢复, 可进一步影响土壤微生物。根据上述 总结和分析可知, 虾蚓对微生物的调控作用主要有 三条途径: 一是直接调控微生物群落; 二是通过改 变化感物质比例调控微生物群落; 三是通过调控土 壤动物区系调控微生物群落。三条途径的综合作用 共同改善了土壤根际微生态, 从而缓解了作物连作 障碍(图2)。

\section{4 展望}

蚯蚓在土壤生态系统中具有重要的作用, 包括 促进有机质分解和养分循环、对土壤理化性质的改 变, 以及与植物、微生物和其他动物的相互作用(张 卫信等, 2007)。与蚳蚓相关的研究涵盖了环境、生 物以及农业等诸多领域。在农业领域, 关于蝶蚓的 研究主要集中在改良污染土壤(Natal-da-Luz et al, 2012; Cao et al, 2016)、影响土壤温室气体排放(Wu et al, 2015; Zhu et al, 2016)、促进土壤养分循环(Cao et al, 2016; Angst et al, 2017)及防控植物病虫害 (Loranger-Merciris et al, 2012; Hume et al, 2015)等方 面, 而通过虹蚓调控土壤系统改良连作土壤的研究 相对较少。结合植物连作障碍发生的过程和现有关 于蝶蚓作用的研究, 笔者认为虾蚓具有缓解连作障 碍的潜力。这一作用主要是通过调控微生物实现 的。近年来, 分子生物学技术的发展为土壤中不可 培养微生物的研究提供了新工具, 提升了人们对土 壤微生物研究的深度和精度, 有关蚯蚂与微生物关 系的研究逐渐增加。蛏蚂一方面可以通过取食、掘 洞和爬行等行为直接影响微生物群落; 另一方面还 可以通过改变动物区系和化感物质间接调控微生 物群落。根据笔者对文献的总结, 目前关于蝶蚂对 微生物直接作用的研究较多(Li et al, 2002; Dempsey et al, 2011; Wu et al, 2012; 曹佳等, 2015; Barbosa et al, 2017), 而通过改变动物区系和化感物质间接影 响微生物的研究较少 (Ilieva-Makulec \& Makulec,
2002; Bi et al, 2018)。蚯蚓既是分解者又是消费者, 其对微生物的间接调控作用是不可忽视的。

植物连作造成化感物质多样性的下降，不仅会 导致土壤微生物群落失衡, 而且会直接影响到植物 的生长。研究表明, 以酚酸为代表的化感物质在一 定条件下不仅抑制植物的发芽和生长，还会抑制植 物的光合、呼吸等生理活动, 使植物抗病能力下降 (Jilani et al, 2008)。因此, 研究虾蚓与连作土壤中化 感物质的关系, 以及䗒蚓加速某些化感物质降解的 机理, 对调控连作土壤环境具有重要意义。

同样，连作造成的土壤动物区系失衡，尤其在 植物根际区，也会直接影响植物。例如某些作物连 作后会造成根结线虫的增加(时立波等, 2010), 根结 线虫不但可对根系造成直接破坏，还能使根系更容 易受到病原菌侵染, 加重植物的连作病害(Mota et $\mathrm{al}, 2013)$ 。而目前关于蛏蚓与土壤动物区系关系的 研究也相对较少。

必须指出, 作物连作障碍的问题是植物进化过 程中产生的一种自然现象，对植物自身而言，有利 于避免近亲繁殖，提高自身的适合度(fitness); 对于 生态系统而言，有利于提高生物多样性。而在农业 生态系统中, 人们为了获得特定的产品, 某些时候 不得不进行重茬种植。因此, 充分发挥土壤生态系 统的自修复作用以及利用土壤食物网的相互作用 关系以减少连作障碍对作物和土壤的影响，是农业 可持续发展的有效途径。

\section{参考文献}

Abbasi MK, Tahir MM, Sabir N, Khurshid M (2015) Impact of the addition of different plant residues on nitrogen mineralization-immobilization turnover and carbon content of a soil incubated under laboratory conditions. Solid Earth, 6, 197-205.

Akca I, Yilmaz NDK, Kizilkaya R (2014) Evaluation of suppression of rhizomania disease by earthworm (Lumbricus terrestris L.) and its effects on soil microbial activity in different sugar beet cultivars. Archives of Agronomy and Soil Science, 60, 1565-1575.

Andriuzzi WS, Phuong-Thi N, Geisen S, Keith AM, Dumack K, Bolger T, Bonkowski M, Brussaard L, Faber JH, Chabbi A, Rumpel C, Schmidt O (2016) Organic matter composition and the protist and nematode communities around anecic earthworm burrows. Biology and Fertility of Soils, 52, 91-100.

Angst Š, Mueller CW, Cajthaml T, Angst G, Lhotáková Z, 
Bartuška M, Špaldoňová A, Frouz J (2017) Stabilization of soil organic matter by earthworms is connected with physical protection rather than with chemical changes of organic matter. Geoderma, 289, 29-35.

Bais HP, Weir TL, Perry LG, Gilroy S, Vivanco JM (2006) The role of root exudates in rhizosphere interactions with plants and other organisms. Annual Review of Plant Biology, 57, 233-266.

Barbosa JZ, Demetrio WC, Silva CM, Dionísio JA (2017) Earthworms (Amynthas spp.) increase common bean growth, microbial biomass, and soil respiration. Semina: Ciências Agrárias, 38, 2887-2898.

Berendsen RL, Pieterse CM, Bakker PA (2012) The rhizosphere microbiome and plant health. Trends in Plant Science, 17, 478-486.

Berg G, Opelt K, Zachow C, Lottmann J, Gotz M, Costa R, Smalla K (2006) The rhizosphere effect on bacteria antagonistic towards the pathogenic fungus Verticillium differs depending on plant species and site. FEMS Microbiology Ecology, 56, 250-261.

Bi YM (2016) Effect and Mechanism for Earthworm to Remit Continuous Cropping Obstacle of Strawberry. PhD dissertation, China Agricultural University, Beijing. (in Chinese with English abstract) [毕艳孟 (2016) 蚯蚂缓解草莓连作 障碍的效应及机理研究. 博士学位论文, 中国农业大学, 北京.]

Bi YM, Tian GL, Wang C, Feng CL, Zhang Y, Zhang LS, Sun ZJ (2016) Application of leaves to induce earthworms to reduce phenolic compounds released by decomposing plants. European Journal of Soil Biology, 75, 31-37.

Bi YM, Tian GL, Wang C, Zhang Y, Wang DN, Zhang FF, Zhang LS, Sun ZJ (2018) Differential effects of two earthworm species on fusarium wilt of strawberry. Applied Soil Ecology, 126, 174-181.

Blouin M, Zuily-Fodil Y, Pham-Thi AT, Laffray D, Reversat G, Pando A, Tondoh J, Lavelle P (2005) Belowground organism activities affect plant aboveground phenotype, inducing plant tolerance to parasites. Ecology Letters, 8, 202-208.

Bonkowski M, Griffiths BS, Ritz K (2000) Food preferences of earthworms for soil fungi. Pedobiologia, 44, 666-676.

Bonkowski M, Schaefer M (1997) Interactions between earthworms and soil protozoa: A trophic component in the soil food web. Soil Biology \& Biochemistry, 29, 499-502.

Boyer J, Reversat G, Lavelle P, Chabanne A (2013) Interactions between earthworms, and plant-parasitic nematodes. European Journal of Soil Biology, 59, 43-47.

Brussaard L, de Ruiter PC, Brown GG (2007) Soil biodiversity for agricultural sustainability. Agriculture Ecosystems \& Environment, 121, 233-244.

Burtelow AE, Bohlen PJ, Groffman PM (1998) Influence of exotic earthworm invasion on soil organic matter, microbial biomass and denitrification potential in forest soils of the northeastern United States. Applied Soil Ecology, 9, 197-202.

Butenschoen O, Ji R, Schaffer A, Scheu S (2009) The fate of catechol in soil as affected by earthworms and clay. Soil Biology \& Biochemistry, 41, 330-339.

Cao J, Wang C, Huang Y, Ji DG, Lou Y (2015) Effects of earthworm on soil microbes and biological fertility: A review. Chinese Journal of Applied Ecology, 26, 1579-1586. (in Chinese with English abstract) [曹佳, 王冲, 皇彦, 纪 丁戈, 楼屹 (2015) 蚳蚓对土壤微生物及生物肥力的影 响研究进展. 应用生态学报, 26, 1579-1586.]

Cao J, Wang C, Ji D (2016) Improvement of the soil nitrogen content and maize growth by earthworms and arbuscular mycorrhizal fungi in soils polluted by oxytetracycline. Science of the Total Environment, 571, 926-934.

Chapuis-Lardy L, Brauman A, Bernard L, Pablo AL, Toucet J, Mano MJ, Weber L, Brunet D, Razafimbelo T, Chotte JL, Blanchart E (2010) Effect of the endogeic earthworm Pontoscolex corethrurus on the microbial structure and activity related to $\mathrm{CO}_{2}$ and $\mathrm{N}_{2} \mathrm{O}$ fluxes from a tropical soil (Madagascar). Applied Soil Ecology, 45, 201-208.

Chen XY, Liu MQ, Hu F, Mao XF, Li HX (2007) Contributions of soil micro-fauna (protozoa and nematodes) to rhizosphere ecological functions. Acta Ecologica Sinica, 27, 3132-3143. (in Chinese with English abstract) [陈小云, 刘 满强, 胡锋, 毛小芳, 李辉信 (2007) 根际微型土壤动物 一原生动物和线虫的生态功能. 生态学报, 27 , 3132-3143.]

Chung EJ, Hossain MT, Khan A, Kim KH, Jeon CO, Chung YR (2015) Bacillus oryzicola sp. nov., an endophytic bacterium isolated from the roots of rice with antimicrobial, plant growth promoting, and systemic resistance inducing activities in rice. Plant Pathology Journal, 31, 152-164.

Corno G, Jürgens K (2006) Direct and indirect effects of protist predation on population size structure of a bacterial strain with high phenotypic plasticity. Applied \& Environmental Microbiology, 72, 78-86.

Dai JJ, Zhang C, Zhou B, Sun YT, Huang YT, Ren ZL, Dai J (2015) Effects of earthworm gut on microbial community structure in heavy metal contaminated soils. Journal of China Agricultural University, 20, 95 - 102. (in Chinese with English abstract) [代金君, 张池, 周波, 孙迎蹈, 黄 钰婷, 任宗玲, 戴军 (2015) 蝶蚓肠道对重金属污染土壤 微生物群落结构的影响. 中国农业大学学报, 20, 95-102.]

Dempsey MA, Fisk MC, Fahey TJ (2011) Earthworms increase the ratio of bacteria to fungi in northern hardwood forest soils, primarily by eliminating the organic horizon. Soil Biology \& Biochemistry, 43, 2135-2141.

Doran JW, Sarrantonio M, Liebig MA (1996) Soil health and sustainability. In: Advances in Agronomy (ed. Sparks DL), pp. 1-54. Elsevier Academic Press Inc., San Diego.

Doran JW, Zeiss MR (2000) Soil health and sustainability: Managing the biotic component of soil quality. Applied Soil 
Ecology, 15, 3-11.

Drake HL, Horn MA (2007) As the worm turns: The earthworm gut as a transient habitat for soil microbial biomes. Annual Review of Microbiology, 61, 169-189.

Du XF , Li YB , Liu F, Su XL, Li Q (2018) Structure and ecological functions of soil micro-food web. Chinese Journal of Applied Ecology, 29, 403-411. (in Chinese with English abstract) [杜晓芳, 李英滨, 刘芳, 宿晓琳, 李琪 (2018) 土壤 微食物网结构与生态功能. 应用生态学报, 29, 403-411.]

Edwards CA, Bohlen PJ (1996) Biology and Ecology of Earthworm, 3rd edn. Chapman and Hall, Springer, London.

Edwards CA, Fletcher KE (1988) Interactions between earthworms and microorganisms in organic-matter breakdown. Agriculture Ecosystems \& Environment, 24, 235-247.

Eisenhauer N, Schlaghamerský J, Reich PB, Frelich LE (2011) The wave towards a new steady state: Effects of earthworm invasion on soil microbial functions. Biological Invasions, 13, 2191-2196.

Elmer WH (2009) Influence of earthworm activity on soil microbes and soilborne diseases of vegetables. Plant Disease, 93, 175-179.

Elmer WH, Ferrandino FJ (2009) Suppression of Verticillium wilt of eggplant by earthworms. Plant Disease, 93, 485-489.

Fang XL, Kuo J, You MP, Finnegan PM, Barbetti MJ (2012) Comparative root colonisation of strawberry cultivars Camarosa and Festival by Fusarium oxysporum f. sp. fragariae. Plant and Soil, 358, 71-85.

Gao MX, Taylor MK, Callaham MA (2017) Trophic dynamics in a simple experimental ecosystem: Interactions among centipedes, Collembola and introduced earthworms. Soil Biology \& Biochemistry, 115, 66-72.

Garbeva P, van Veen JA, van Elsas JD (2004) Microbial diversity in soil: Selection microbial populations by plant and soil type and implications for disease suppressiveness. Annual Review of Phytopathology, 42, 243-270.

Geisen S, Koller R, Hünninghaus M, Dumack K, Urich T, Bonkowski M (2016) The soil food web revisited: Diverse and widespread mycophagous soil protists. Soil Biology \& Biochemistry, 94, 10-18.

Gomez-Brandon M, Aira M, Lores M, Dominguez J (2011) Epigeic earthworms exert a bottleneck effect on microbial communities through gut associated processes. PLoS ONE, 6, e24786.

González G, Seastedt TR (2001) Soil fauna and plant litter decomposition in tropical and subalpine forests. Ecology, 82, 955-964.

Gopalakrishnan S, Pande S, Sharma M, Humayun P, Kiran BK, Sandeep D, Vidya MS, Deepthi K, Rupela O (2011) Evaluation of actinomycete isolates obtained from herbal vermicompost for the biological control of Fusarium wilt of chickpea. Crop Protection, 30, 1070-1078.

Gyaneshwar P, Kumar GN, Parekh LJ, Poole PS (2003) Role of soil microorganisms in improving $\mathrm{P}$ nutrition of plants. Sys- tem Sciences \& Comprehensive Studies in Agriculture, 245, 83-93.

He JZ, Ge Y, Xu ZH, Chen CR (2009) Linking soil bacterial diversity to ecosystem multifunctionality using backward-elimination boosted trees analysis. Journal of Soils and Sediments, 9, 547-554.

Hu YX, Sun ZJ, Zhou FY, Liu XY, Cao AC (2002) Study on suppressness effect of vermicompost to soil-borne disease of cucumber seedlings. Acta Ecologica Sinica, 22, 1106-1115. (in Chinese with English abstract) [胡艳霞, 孙振钧, 周法 永, 刘小秧, 曹坳程 (2002) 蝶蚓粪对黄瓜苗期土传病害 的抑制作用. 生态学报, 22, 1106-1115.]

Huang K, Li FS, Wei YF, Chen XM, Fu XY (2013a) Changes of bacterial and fungal community compositions during vermicomposting of vegetable wastes by Eisenia foetida. Bioresource Technology, 150, 235-241.

Huang LF, Song LX, Xia XJ, Mao WH, Shi K, Zhou YH, Yu JQ (2013b) Plant-soil feedbacks and soil sickness: From mechanisms to application in agriculture. Journal of Chemical Ecology, 39, 232-242.

Hume EA, Horrocks AJ, Fraser PM, Curtin D, Meenken ED, Chng S, Beare MH (2015) Alleviation of take-all in wheat by the earthworm Aporrectodea caliginosa (Savigny). Applied Soil Ecology, 90, 18-25.

Ilieva-Makulec K, Makulec G (2002) Effect of the earthworm Lumbricus rubellus on the nematode community in a peat meadow soil. European Journal of Soil Biology, 38, 59-62.

Janvier C, Villeneuve F, Alabouvette C, Edel-Hermann V, Mateille T, Steinberg C (2007) Soil health through soil disease suppression: Which strategy from descriptors to indicators? Soil Biology \& Biochemistry, 39, 1-23.

Jilani G, Mahmood S, Chaudhry AN, Hassan I, Akram M (2008) Allelochemicals: Sources, toxicity and microbial transformation in soil-A review. Annals of Microbiology, 58, 351-357.

Jing ZW, Chen RR, Wei SP, Feng YZ, Zhang JB, Lin XG (2017) Response and feedback of C mineralization to $P$ availability driven by soil microorganisms. Soil Biology \& Biochemistry, 105, 111-120.

Jones CG, Lawton JH, Shachak M (1994) Organisms as ecosystem engineers. Oikos, 69, 373-386.

Kim J-S, Lee J, Lee C-H, Woo SY, Kang H, Seo S-G, Kim S-H (2015) Activation of Pathogenesis-related genes by the rhizobacterium, Bacillus sp. JS, which induces systemic resistance in tobacco plants. Plant Pathology Journal, 31, 195-201.

Koike ST, Kirkpatrick SC, Gordon TR (2009) Fusarium wilt of strawberry caused by Fusarium oxysporum in California. Plant Disease, 93, 1077.

Kulmatiski A, Beard KH, Stevens JR, Cobbold SM (2008) Plant-soil feedbacks: A meta-analytical review. Ecology Letters, 11, 980-992.

Lambers H, Mougel C, Jaillard B, Hinsinger P (2009) 
Plant_microbe_soil interactions in the rhizosphere: An evolutionary perspective. Plant and Soil, 321, 83-115.

Larkin RP (2015) Soil health paradigms and implications for disease management. Annual Review of Phytopathology, 53, 199-221.

Lee KE (1985) Earthworms, Their Ecology and Relationships with Soils and Land Use, Academic Press, Sydney.

Li H, Du ZY, Liu Q, Shi YX (2016) Effect of earthworm-mycorrhiza interaction on soil enzyme activities, root growth and nutrients uptake of sweet potato. Journal of Plant Nutrition \& Fertilizer, 22, 209-215. (in Chinese with English abstract) [李欢, 杜志勇, 刘庆, 史衍胥 (2016) 蝶蚓 菌根互作对土壤酶活、甘薯根系生长及养分吸收的影响. 植物营养与肥料学报, 22, 209-215.]

Li HQ, Liu QZ, Zhang LL, Wang YL, Zhang H, Bai PH, Luan XB (2014) Accumulation of phenolic acids in the monocultured strawberry soils and their effect on soil nematodes. Chinese Journal of Ecology, 33, 169-175. (in Chinese with English abstract) [李贺勤, 刘奇志, 张林林, 王玉玲, 张 航, 白鹏华, 奕小兵 (2014) 草莓连作土壤酚酸类物质积 累对土壤线虫的影响. 生态学杂志, 33, 169-175.]

Li X, Fisk MC, Fahey TJ, Bohlen PJ (2002) Influence of earthworm invasion on soil microbial biomass and activity in a northern hardwood forest. Soil Biology \& Biochemistry, 34, 1929-1937.

Li XG, Ding CF, Zhang TL, Wang XX (2014) Fungal pathogen accumulation at the expense of plant-beneficial fungi as a consequence of consecutive peanut monoculturing. Soil Biology \& Biochemistry, 72, 11-18.

Lipiec J, Frąc M, Brzezińska M, Turski M, Oszust K (2016) Linking microbial enzymatic activities and functional diversity of soil around earthworm burrows and casts. Frontiers in Microbiology, 7, 1361.

Liu X, Zhang JL, Gu TY, Zhang WM, Shen QR, Yin SX, Qiu HZ (2014) Microbial community diversities and taxa abundances in soils along a seven-year gradient of potato monoculture using high throughput pyrosequencing approach. PLoS ONE, 9, e86610.

Loranger-Merciris G, Cabidoche YM, Deloné B, Quénéhervé P, Ozier-Lafontaine H (2012) How earthworm activities affect banana plant response to nematodes parasitism. Applied Soil Ecology, 52, 1-8.

Marinissen JCY, Bok J (1988) Earthworm-amended soil structure: Its influence on Collembola population in grassland. Pedobiologia, 32, 243-252.

Mclean MA, Parkinson D (1998) Impacts of the epigeic earthworm Dendrobaena octaedra on oribatid mite community diversity and microarthropod abundances in pine forest floor: A mesocosm study. Applied Soil Ecology, 7, 125-136.

Mohan KV, Hrushikesh N, Sreehari K, Kumar TA, Vidyavathi N, Pallavi A (2011) Studies on bioremediation of phenol by earthworm. International Journal of Environmental Sciences, 1, 1268-1273.
Moore JC, Zwetsloot HJC, Ruiter PCD (1990) Statistical analysis and simulation modelling of the belowground food webs of two winter wheat management practices. Netherlands Journal of Agricultural Science, 38, 303-316.

Mota FC, Alves GCS, Giband M, Gomes ACMM, Sousa FR, Mattos VS, Barbosa VHS, Barroso PAV, Nicole M, Peixoto JR, Rocha MR, Carneiro RMDG (2013) New sources of resistance to Meloidogyne incognita race 3 in wild cotton accessions and histological characterization of the defence mechanisms. Plant Pathology, 62, 1173-1183.

Nannipieri P, Ascher J, Ceccherini MT, Landi L, Pietramellara G, Renella G (2010) Microbial diversity and soil functions. European Journal of Soil Science, 54, 655-670.

Natal-da-Luz T, Lee I, Verweij RA, Morais PV, Van Velzen MJ, Sousa JP, Van Gestel CA (2012) Influence of earthworm activity on microbial communities related with the degradation of persistent pollutants. Environmental Toxicology and Chemistry, 31, 794-803.

Niu XQ, Li JH, Zhang JL, Shen BY, Chai ZX, Wang D (2011) Changes of Fusarium in rhizosphere soil under potato continuous cropping systems in arid-irrigated area of Gansu Province. Acta Prataculturae Sinica, 20, 236-243.

Oka Y (2010) Mechanisms of nematode suppression by organic soil amendments-A review. Applied Soil Ecology, 44, 101-115.

Oldenburg E, Kramer S, Schrader S, Weinert J (2008) Impact of the earthworm Lumbricus terrestris on the degradation of Fusarium-infected and deoxynivalenol-contaminated wheat straw. Soil Biology \& Biochemistry, 40, 3049-3053.

Salmon S, Ponge JF (2001) Earthworm excreta attract soil springtails: Laboratory experiments on Heteromurus nitidus (Collembola: Entomobryidae). Soil Biology \& Biochemistry, 33, 1959-1969.

Sampedro L, Whalen JK (2007) Changes in the fatty acid profiles through the digestive tract of the earthworm Lumbricus terrestris L. Applied Soil Ecology, 35, 226-236.

Sauvadet M, Chauvat M, Cluzeau D, Maron PA, Villenave C, Bertrand I (2016) The dynamics of soil micro-food web structure and functions vary according to litter quality. Soil Biology \& Biochemistry, 95, 262-274.

Schaefer M, Petersen SO, Filser J (2005) Effects of Lumbricus terrestris, Allolobophora chlorotica and Eisenia fetida on microbial community dynamics in oil-contaminated soil. Soil Biology \& Biochemistry, 37, 2065-2076.

Schrader S, Wolfarth F, Oldenburg E (2013) Biological control of soil-borne phytopathogenic fungi and their mycotoxins by soil fauna-A review. Bulletin of the University of Agricultural Sciences \& Veterinary, 70, 291-298.

Shan J, Liu J, Wang YF, Yan XY, Guo HY, Li XZ, Ji R (2013) Digestion and residue stabilization of bacterial and fungal cells, protein, peptidoglycan, and chitin by the geophagous earthworm Metaphire guillelmi. Soil Biology \& Biochemistry, 64, 9-17. 
Shi LB, Wang ZH, Wu HY, Liu J (2010) Influence of continuous tomato-cropping on second-stage juveniles of root-knot nematode and free-living nematodes from rhizosphere soil in plastic greenhouse. Acta Phytopathologica Sinica, 40, 81-89. (in Chinese with English abstract) [时立波, 王振华, 吴海燕, 刘静 (2010) 连作年限对番茄根围土壤根结线 虫二龄幼虫与自由生活线虫数量的影响. 植物病理学报, 40, 81-89.]

Singh JS, Raghubanshi AS, Singh RS, Srivastava SC (1989) Microbial biomass acts as a source of plant nutrients in dry tropical forest and savanna. Nature, 338, 499-500.

Song YY, Zeng RS, Xu JF, Li J, Shen X, Yihdego WG (2010) Interplant communication of tomato plants through underground common mycorrhizal networks. PLoS ONE, 5, e13324.

Tao J, Chen XY, Liu MQ, Hu F, Griffiths B, Li HX (2009) Earthworms change the abundance and community structure of nematodes and protozoa in a maize residue amended rice-wheat rotation agro-ecosystem. Soil Biology \& Biochemistry, 41, 898-904.

Tao J, Xu YJ, Griffiths BS, Hu F, Chen XY, Jiao JG, Li HX (2011) Earthworms reduce the abundance of nematodes and enchytraeids in a soil mesocosm experiment despite abundant food resources. Soil Science Society of America Journal, 75, 1774-1778.

Teste FP, Kardol P, Turner BL, Wardle DA, Zemunik G, Renton M, Laliberte E (2017) Plant-soil feedback and the maintenance of diversity in Mediterranean-climate shrublands. Science, 355, 173-176.

Thakuria D, Schmidt O, Finan D, Egan D, Doohan FM (2010) Gut wall bacteria of earthworms: A natural selection process. The ISME Journal, 4, 357-366.

Toljander JF, Lindahl BD, Paul LR, Elfstrand M, Finlay RD (2007) Influence of arbuscular mycorrhizal mycelial exudates on soil bacterial growth and community structure. FEMS Microbiology Ecology, 61, 295-304.

Vaitauskiene K, Sarauskis E, Naujokiene V, Liakas V (2015) The influence of free-living nitrogen-fixing bacteria on the mechanical characteristics of different plant residues under no-till and strip-till conditions. Soil \& Tillage Research, 154, 91-102.

Watkins AJ, Nicol GW, Shaw LJ (2009) Use of an artificial root to examine the influence of 8-hydroxyquinoline on soil microbial activity and bacterial community structure. Soil Biology \& Biochemistry, 41, 580-585.

Wolfarth F, Schrader S, Oldenburg E, Weinert J (2011) Contribution of the endogeic earthworm species Aporrectodea caliginosa to the degradation of deoxynivalenol and Fusarium biomass in wheat straw. Mycotoxin Research, 27, 215-220.

Wu FY, Wan JHC, Wu SC, Wong MH (2012) Effects of earthworms and plant growth-promoting rhizobacteria (PGPR) on availability of nitrogen, phosphorus, and potas- sium in soil. Journal of Plant Nutrition and Soil Science, 175, 423-433.

Wu JH, Song CY, Chen JK (2007) Effect of microbivorous nematodes on plant growth and soil nutrient cycling: A review. Biodiversity Science, 15, 124-133. (in Chinese with English abstract) [吴纪华, 宋慈玉, 陈家宽 (2007) 食微 线虫对植物生长及土壤养分循环的影响. 生物多样性, 15, 124-133.]

Wu YP, Lü LY, Bi YM, Zhang Y, Sun ZJ (2013) Effects of earthworm inoculation on saline-alkali soil nutrient, soil organisms and plant cultivation. Journal of China Agricultural University, 18, 45-51. (in Chinese with English abstract) [伍玉鹏, 吕丽媛, 毕艳孟, 张一, 孙振钧 (2013) 接种蝶 蚓对盐碱土养分、土壤生物及植被的影响. 中国农业大学 学报, 18, 45-51.]

Wu YP, Muhammad S, Zhao JS, Hao R, Hu RG (2015) Effect of the earthworm gut-stimulated denitrifiers on soil nitrous oxide emissions. European Journal of Soil Biology, 70, 104-110.

Wu ZW, Wang MD, Liu XY, Chen HG, Jia XC (2009) Phenolic compounds accumulation in continuously cropped Rehmannia glutinosa soil and their effects on $R$. glutinosa growth. Chinese Journal of Ecology, 28, 660-664. (in Chinese with English abstract) [吴宗伟, 王明道, 刘新育, 陈 红歌, 贾新成 (2009) 重茬地黄土壤酚酸的动态积累及 其对地黄生长的影响. 生态学杂志, 28, 660-664.]

Wurst S, De Devn GB, Orwin K (2013) Soil biodiversity and functions. In: Soil Ecology and Ecosystem Services (eds Wall DH, Bardgett RD, Behan-Pelletier V, Herrick JE, Jones TH, Six J, Strong DR, van der Putten WH), pp. 28-45. Oxford University Press, Oxford.

Yahaya ANA, Hossain MS, Edyvean R (2017) Analysis of phenolic compounds in empty fruit bunches in oyster mushroom cultivation and in vermicomposting. BioResource, 12, 4594-4605.

Yao Y, He J, Zhang Y, Li YF, Wu YP (2015) Effects of Eisenia fetida inoculation on soil organic carbon and soil microorganisms under rice straw application. Journal of Agro-Environment Science, 34, 110-117. (in Chinese with English abstract) [姚影, 何静, 张一, 李钰飞, 伍玉鹏 (2015) 赤子爱胜蚓(Eisenia fetida) 对秸秆施入后土壤有机 碳和微生物的影响. 农业环境科学学报, 34, 110-117.]

Ye SF, Yu JQ, Peng YH, Zheng JH, Zou LY (2004) Incidence of Fusarium wilt in Cucumis sativus L. is promoted by cinnamic acid, an autotoxin in root exudates. Plant and Soil, 263, 143-150.

Zhang BG, Li GT, Shen TS, Wang JK, Sun Z (2000) Changes in microbial biomass $\mathrm{C}, \mathrm{N}$, and $\mathrm{P}$ and enzyme activities in soil incubated with the earthworms Metaphire guillelmi or Eisenia fetida. Soil Biology \& Biochemistry, 32, 2055-2062.

Zhang N, Wang DD, Liu YP, Li SQ, Shen QR, Zhang RF (2014) Effects of different plant root exudates and their or- 
ganic acid components on chemotaxis, biofilm formation and colonization by beneficial rhizosphere-associated bacterial strains. Plant and Soil, 374, 689-700.

Zhang WW, Cao J, Zhang SD, Wang C (2016) Effect of earthworms and arbuscular mycorrhizal fungi on the microbial community and maize growth under salt stress. Applied Soil Ecology, 107, 214-223.

Zhang WX, Chen DM, Zhao CC (2007) Functions of earthworm in ecosystem. Biodiversity Science, 15, 142-153. (in Chinese with English abstract) [张卫信, 陈迪马, 赵灿灿 (2007) 蚳蚓在生态系统中的作用. 生物多样性, 15, 142-153.]

Zhang XP, Hou WL, Chen P (2001) Soil animal guilds and their ecological distribution in the northeast of China. Chinese Journal of Applied \& Environmental Biology, 7, 370-374. (in Chinese with English abstract) [张雪萍, 侯威 岭, 陈鹏 (2001) 东北森林土壤动物同功能种团及其生 态分布. 应用与环境生物学报, 7, 370-374.]

Zhang Y, Wang GC, Wu YP, Zhao H, Zhang YF, Sun ZJ (2013) PCR-DGGE analysis of earthworm gut bacteria diversity in stress of Escherichia coli O157: H7. Advances in Bioscience and Biotechnology, 4, 437-441.

Zhang Y, Zhang DJ, Li X, Zhang J (2018) Contribution of soil fauna to the degradation of recalcitrant components in Cinnamomum camphora foliar litter in different-sized gaps in Pinus massoniana plantations. Journal of Forestry Research. https://doi.org/10.1007/s11676-018-0609-6.

Zhen WC, Dai L, Hu TL, Cao KQ (2005) Study on dynamics of soil microorganisms under strawberry (Fragria ananassa Duch) continuous cropping. Journal of Agricultural University of Hebei, 28, 70-72, 87. (in Chinese with English abstract) [甄文超, 代丽, 胡同乐, 曹克强 (2005) 连作草莓 土壤微生物区系动态的研究. 河北农业大学学报, 28, 70-72, 87.]

Zheng XQ, Fan XF, Zhang HL, Li SX, Wang JQ, Zhang JQ, Wang LJ, Tao XB, Lü WG (2015) Effects of Pheretima guillelmi cultivation time on microbial community diversity and characteristics of carbon metabolism in vegetable soil. Journal of Agricultural Resources \& Environment, 32, 596 - 602. (in Chinese with English abstract) [郑宪清, 范晓芬, 张翰林, 李双喜, 王金庆, 张娟琴, 王良军, 陶晓斌, 吕卫光 (2015) 威廉环毛蚓耕作时间对菜田土壤微生物 群落多样性及碳代谢特征的影响. 农业资源与环境学报, 32, 596-602.]

Zhu XY, Chang L, Liu J, Zhou MH, Li JJ, Gao B, Wu DH (2016) Exploring the relationships between soil fauna, different tillage regimes and $\mathrm{CO}_{2}$ and $\mathrm{N}_{2} \mathrm{O}$ emissions from black soil in China. Soil Biology \& Biochemistry, 103, 106-116.

Zhu YH, Li KZ, Lu L (2012) Rhizosphere soil fauna and its effects on plant growth: A review. Chinese Journal of Ecology, 31, 2688-2693. (in Chinese with English abstract) [朱 永恒, 李克中, 陆林 (2012) 根际土壤动物及其对植物生 长的影响. 生态学杂志, 31, 2688-2693.]

(责任编委: 傅声雷 责任编辑: 时意专) 